\title{
The value of anticarcinoembryonic antigen, human milk factor globulin, and antikeratin antibodies in differentiating mesothelioma from lung carcinoma
}

\author{
V M Joglekar, D Oliver, $M$ Harris
}

\begin{abstract}
Monoclonal anticarcinoembryonic antigen (antiCEA), human milk factor globulin (HMFG2), and antikeratin antibodies were assessed for their value in the differential diagnosis of pleural mesothelioma (53 cases) and carcinoma of the lung (60 cases) in material from necropsies. In 40 of the cases pleural biopsies were also studied in the same manner. AnticEA was found to be the best discriminating antibody for most types of mesothelioma; HMFG2 was slightly less valuable but a useful additional tool. Antikeratin was the least useful. For both antiCEA and HMFG2 antibodies, however, the proportion of carcinomas staining was smaller than in previous studies and this, combined with the positive staining of some mesotheliomas, reduces the value of the reactions in the individual case. Medical panels adjudicating compensation claims should not use these reactions as the sole criteria in deciding the origin of the tumours in these cases.
\end{abstract}

The problem of differentiating pleural mesothelioma from lung carcinoma in histological and cytological material is well known, particularly when mesothelioma cells have an epithelial appearance. Immunological techniques using antiCEA, HMFG, antikeratin, and other antibodies have been employed by several authors on histological ${ }^{1-6}$ and cytological material from effusions..$^{7-11}$ These have been used on their own or together with conventional techniques-for example, PAS \pm diastase (neutral mucins), Alcian blue \pm hyaluronidase (acid mucins), slycosoaminoglycan analysis, and electron micro-

Department of Histopathology, North Lonsdale Hospital, Barrow-in-Furness

V M Joglekar, D Oliver

Christie Hospital, Manchester $M$ Harris scopy. None of these techniques has proved diagnostic on its own. Immunological studies initially gave conflicting results with antiCEA, ${ }^{13}$ but recent studies $^{689}$ show antiCEA to be negative in most cases of mesothelioma. Conflicting ${ }^{36}$ or equivocal results ${ }^{5}$ have been reported for HMFG2.

Antikeratin ${ }^{369}$ shows a positive reaction in most cases of mesothelioma but it is not useful in practice as many adenocarcinomas also show a similar reaction. In this study we have assessed the value of three commonly used antibodies-namely, antiCEA, HMFG2, and antikeratin (AE1/AE3) in a series of postmortem cases of pleural mesothelioma and carcinoma of the lung with pleural involvement. Many of the cases of lung carcinoma closely mimicked mesothelioma clinically and radiologically, reproducing the situation faced by pathologists dealing with biopsies or necropsies in compensation cases in which there is a real diagnostic dilemma. Also in some of the cases biopsy material from the same patients was studied and compared with the findings from necropsy tissue.

\section{Materials and methods}

Fifty three cases of mesothelioma and 60 cases of lung carcinoma, diagnosed between 1979 and 1987, were extracted from the postmortem files in the Department of Pathology at North Lonsdale Hospital. Fifty eight of these cases (34 mesotheliomas, 24 carcinomas) had undergone pleural biopsy, the rest presenting terminally. In all of the 113 cases the postmortem material was further examined by pathologists at the local pneumoconiosis medical panel. In most cases they concurred with our original diagnosis but when they disagreed the panel opinion was accepted for our analysis.

\section{SPECIMEN PROCESSING}

Paraffin sections $(4 \mu)$ from the 60 pleural biopsies and 113 postmortem tumour blocks were routinely stained with haematoxylin and eosin. They were surveyed to ensure that tumour cells were present for staining. In the case of 12 pleural biopsies no malignant cells were seen and these were excluded from the study. 
Further $4 \mu$ sections were cut for the alkaline phosphatase antialkaline phosphatase (APAAP) immunostaining technique. Eight pleural biopsy cases now had insufficient cells for immunostaining and these were excluded from the study leaving 40 pleural biopsies for analysis (13 carcinomas, 17 mesotheliomas). The sections were labelled numerically by one of us (DO), who was aware of the diagnosis. The sections were mounted with starch adhesive and allowed to dry for 30 minutes at $60^{\circ} \mathrm{C}$. Sections were treated with trypsin for 20 minutes, stained by the APAAP immunoalkaline staining method as previously described, ${ }^{13}$ and mounted with glycerine jelly.

The stained sections were then assessed independently and blindly by two of us (VMJ, MH). Staining was considered positive when most cells showed the reddish reaction product or negative if no reaction was observed. The two assessments were then correlated with each other and with the pleural biopsy and postmortem diagnosis at the end of the study.

\section{REAGENTS}

The antibodies AE1/AE3 (low molecular weight antikeratin mouse monoclonal antibody), HFMG2 (supernatant), and antiCEA mouse monoclonal antibodies were supplied by Oxoid Ltd, Wade Road, Basingstoke, Hampshire RG24 OPW. The antiCEA antibody had not been absorbed with spleen powder to remove immunoreactive activity to non-specific reaction antigen.

Positive controls consisted of tumour from colon (antiCEA), skin (antikeratin), and breast (HMFG2). Negative controls were the same but with no antibody.

\section{Results}

Tables 1 and 2 summarise the immunohistochemical results.

\section{Discussion}

In this study we attempted to assess the value of three commonly used antibodies in the differentiation of malignant pleural mesothelioma from carcinoma involving the pleura.

We investigated a series of necropsy cases comprising carcinomas of various histological types, in which the diagnosis had been authenticated by the local pneumoconiosis panel and in which the carcinoma clinically and radiologically mimicked mesothelioma. In 40 of the 113 cases biopsy material was also studied. Postmortem confirmation of the original diagnosis, a useful end point, has not been a feature of some studies, ${ }^{2-6}$ whereas other studies ${ }^{19}$ have attempted to get postmortem confirmation of their diagnosis but did not succeed in all the cases.

No specific tumour markers have yet been recognised for mesothelioma. As this tumour features in any differential diagnosis of lung carcinoma, particularly involving pleura, various authors ${ }^{1-9}$ have used panels of antibodies in an effort to achieve this differentiation. Early studies ${ }^{1-3}$ used polyclonal antibodies but later, as monoclonal antibodies became available, these were used. They included antiCEA, antikeratins (AE1, 2, 3, KL1), EMA, HMFG2, Factor VIII, Leu M1 and CA1, CA2, and CA3. ${ }^{11}$ The immunoperoxidase technique was frequently used ${ }^{13579}$ and Battifora and Kopinski ${ }^{6}$ used an avidine/biotin technique. Later authors ${ }^{11}$ used the APAAP technique on serous effusions mainly because of a better recognisable reaction product compared with immunoperoxidase. The

Table 1 Antibody results for postmortem material from 113 cases

\begin{tabular}{|c|c|c|c|c|c|c|}
\hline \multirow[b]{3}{*}{ Tumour type (No of cases) } & \multicolumn{6}{|c|}{ Antibody reaction } \\
\hline & \multicolumn{2}{|c|}{ Antikeratin AE1 (No (\%)) } & \multicolumn{2}{|c|}{ HMFG $2(N o(\%))$} & \multicolumn{2}{|c|}{$C E A(N o(\%))$} \\
\hline & + & - & + & - & + & - \\
\hline $\begin{array}{l}\text { Mesothelioma (53) } \\
\text { Carcinoma (60) }\end{array}$ & $\begin{array}{l}40(75 \cdot 5) \\
40(66 \cdot 7)\end{array}$ & $\begin{array}{l}13(24 \cdot 5) \\
20(33 \cdot 3)\end{array}$ & $\begin{array}{l}10(18 \cdot 9) \\
29(48 \cdot 3)\end{array}$ & $\begin{array}{l}43(80 \cdot 1) \\
31(51 \cdot 7)\end{array}$ & $\begin{array}{r}2(3 \cdot 8) \\
22(36 \cdot 7)\end{array}$ & $\begin{array}{l}51(96 \cdot 2) \\
38(63 \cdot 3)\end{array}$ \\
\hline
\end{tabular}

Table 2 Antibody results for pleural biopsy material from 40 cases

\begin{tabular}{|c|c|c|c|c|c|c|}
\hline \multirow[b]{3}{*}{ Tumour type (No of cases) } & \multicolumn{6}{|c|}{ Antibody reaction } \\
\hline & \multicolumn{2}{|c|}{ Antikeratin AE1 (No (\%)) } & \multicolumn{2}{|c|}{$H M F G 2(N o(\%))$} & \multicolumn{2}{|c|}{$C E A(N o(\%))$} \\
\hline & + & - & + & - & + & - \\
\hline $\begin{array}{l}\text { Mesothelioma (26) } \\
\text { Carcinoma (14) }\end{array}$ & $\begin{array}{l}17(65.4) \\
11(78.6)\end{array}$ & $\begin{array}{l}9(34 \cdot 6) \\
3(21 \cdot 4)\end{array}$ & $\begin{array}{l}6(23 \cdot 1) \\
6(42 \cdot 6)\end{array}$ & $\begin{array}{r}20(76 \cdot 9) \\
8(57 \cdot 4)\end{array}$ & $\begin{array}{l}4(15 \cdot 4) \\
5(35 \cdot 7)\end{array}$ & $\begin{array}{r}22(84 \cdot 6) \\
9(64 \cdot 3)\end{array}$ \\
\hline
\end{tabular}


APAAP technique has been tried previously on histological material from lung carcinoma and mesothelioma. ${ }^{11}$ In our experience the intensity of the staining reaction did not appear to deteriorate in postmortem material and the red staining reaction was easier to interpret compared with the brown reaction product of the immunoperoxidase method.

\section{NECROPSY STUDY}

AntiCEA

Table 1 shows that antiCEA is by far the most useful antibody, 51 out of $53(96.2 \%)$ mesotheliomas staining negatively. In our study antiCEA was also absent, however, in many cases of carcinoma (38 of 60; $63.3 \%)$. This is a higher percentage of negative carcinomas than in previous reports, ${ }^{1391112}$ which show a range between $0^{12}$ and $28 \% .^{9}$ Initial reports ${ }^{312}$ gave conflicting results, perhaps due to the use of trypsin, different polyclonal antisera, or different staining techniques, or due to non-specific, cross reacting antigen. When this was absorbed by spleen powder and when monoclonal antiCEA with prior trypsin digestion (as in this study) was used, mesotheliomas were usually negative. The occurrence of a high proportion of antiCEA negative adenocarcinomas in our study obviously reduces the value of the reaction as a discriminant between carcinoma and mesothelioma.

\section{HMFG2}

The next most useful antibody was HMFG2, 43 out of $53(80 \cdot 1 \%)$ mesotheliomas staining negatively. Fifty two per cent of carcinomas were also negative, however. Early studies gave differing results with HMFG2; thus Battifora and Kopinski ${ }^{6}$ found negative staining for HMFG2 in mesothelioma (0/16), whereas Marshall et $a l^{\beta}(12 / 16)$ and Stickler et $a l^{10}(6 / 6)$ found positive staining with the same antibody. Our results differ from these studies in that HMFG2 positivity occurred in a minority of mesotheliomas but was more likely to be negative. Conflicting results in earlier studies may have been due to the small sample analysed compared with the amount in our study. A contributary factor may be the affinity of the sera used, as the HMFG2 molecule is a large one with multiple binding sites. ${ }^{2}$ Our results indicate that HMFG2 would be a useful second antibody in the diagnosis of mesothelioma in combination with antiCEA.

\section{Antikeratin}

Although an early report ${ }^{1}$ suggested that antikeratin profiles helped to distinguish adenocarcinoma from mesothelioma, later studies ${ }^{569}$ failed to support this view. A reason for this may have been the use of polyclonal antisera in the early study or the testing of only a small number of mesotheliomas. Our study with a monoclonal antiserum shows that antikeratin antibodies are not useful in distinguishing mesothelioma from lung carcinoma, similar staining reactions occurring in both situations. An immunoperoxidase study ${ }^{14}$ of 30 cases of sarcomatoid, and mixed types of diffuse malignant mesothelioma, however, showed that it helps to distinguish this group from other spindle cell lung neoplasms. Our 10 cases in this group did not provide a result that was as clear cut.

\section{BIOPSY AUTOPSY CORRELATION}

The pleural biopsy part of this study showed that in any individual case the main problem was the inadequate harvest of diagnostic cells; of the original 60 cases 20 had to be eliminated for this reason. When tumour was present in sufficient quantity the antiCEA and HMFG2 reaction correlated reasonably well with the postmortem results. A practical point is that normal alveolar lining cells stain positively for HMFG2 so that care must be taken to ensure that only tumour cells are evaluated in the pleural biopsy. Also leucocytes sometimes stain positively for antiCEA although these were not likely to be confused with tumour cells.

In general the three antibodies showed the same trends found in the postmortem series. When the pleural biopsy series was compared with the postmortem results, complete agreement was found with the three antibodies tested in 26 out of 40 cases ( $65 \%$; 15 mesotheliomas, 11 carcinomas). Discrepancies in the 14 cases between the two series occurred in the antikeratin reactions (11 mesotheliomas, one carcinoma), HMFG2 (five mesotheliomas, one carcinoma), and antiCEA (one mesothelioma, one carcinoma). In every case the discrepancy resulted from a negative biopsy reaction and a positive postmortem reaction suggesting a sampling problem in the pleural biopsy.

The diagnosis of pleural mesothelioma at necropsy examination is an important problem for the pathologist as the success or failure of individual compensation claims can hinge on that person's conclusions. In this study we have shown that in most cases immunohistochemistry on material from necropsy accurately reflects the results obtained in biopsies from the same patients, suggesting that it is a valid diagnostic procedure. Although our results confirm that antiCEA and HMFG2 are useful in distinguishing mesothelioma from carcinoma, we found a smaller proportion of carcinomas in general and adenocarcinomas in particular staining with the two antibodies than most other workers. This, taken together with our finding and those in other series that a number of mesotheliomas are positive for antiCEA and HMFG2 reduces the value of the staining reactions when applied to the individual case. They should not, therefore, be used as the sole criteria by medical panels in deciding the origin of a given tumour for mesothelioma compensation claims. 
We thank the Northern Regional Health Authority research committee for providing the funds to conduct this project.

Requests for reprints to: Dr V M Joglekar, Department of Pathology, Furness General Hospital, Dalton Lane, Barrow-in-Furness, Cumbria LA14 4LF.

1 Corson JM, Pinkus GS. Mesothelioma: profile of keratin proteins and carcinoembryonic antigen. Am $J$ Pathol 1982;108:80-8.

2 Ghosh AK, Spriggs AI, Taylor-Pappadimitrou J, Mason DY. Immunochemical staining of cells in pleural and peritoneal effusions with a panel of monoclonal antibodies. J Clin Pathol 1983;36:1154-64.

3 Marshall RJ, Herbert A, Braye SG, Jones DB. Use of antibodies to carcinoembryonic antigen and human milk factor globulin to distinguish carcinoma, mesothelioma and reactive mesothelium. J Clin Pathol 1984;37:1215-21.

4 Warhol MJ, Corson JM. An ultrastructural comparison of mesotheliomas with adenocarcinomas of the lung and breast. Hum Pathol 1985;16:50-5.

5 Loosli H, Hurlimann J. Immunohistological study of malignant diffuse mesotheliomas of the pleura. Histopathology 1984;8:793-803.
6 Battifora H, Kopinski MI. Distinction of mesothelioma from adenocarcinoma. Cancer 1985;55:1679-85.

$7 \mathrm{Kahn} \mathrm{HJ}$, Thorner PS, Yeger H. Distinct keratin patterns demonstrated by immunoperoxidase staining of adenocarcinomas, carcinoids and mesotheliomas using polyclonal and monoclonal antikeratin antibodies. Am $J$ Clin Pathol 1986;86:556-74.

8 Pinkus GS, Etheridge CL, O'Connor EM. Are keratin proteins a better tumor marker than epithelial membrane antigen? Am J Clin Pathol 1986;85:269-77.

9 Cibas E, Corson J, Pinkus G. The distinction of adenocarcinoma from malignant mesothelioma in cell blocks of effusions. Hum Pathol 1987;18:67-74.

10 Stickler JG, Herndier BG, Rouse RV. Immunohistochemical staining in malignant mesothelioma. Am J Clin Pathol 1987;88:610-4.

11 Ghosh AK, Gatter KC, Dunnill MS, Mason DY. Immunohistological staining of reactive mesothelium, mesothelioma and lung carcinoma with a panel of monoclonal antibodies. J Clin Pathol 1987;40:19-25.

12 Holden J, Churg A. Immunohistochemical staining for keratin and carcinoembryonic antigen in the diagnosis of malignant mesothelioma. Am J Surg Pathol 1984;8:277-9.

13 Cordell JL, Falini B, Erber WN, et al. Immunoenzymatic labelling of monoclonal antibodies using immune complexes of alkaline phosphatase and monoclonal antialkaline phosphatase (APAAP) complexes. J Histochem Cytochem 1984;32:219-29.

14 Montag AG, Pinkus GS, Corson JM. Keratin protein immunoreactivity of sarcomatoid and mixed types of diffuse and malignant mesothelioma: An immunoperoxidase study of 30 cases. Hum Pathol 1988;19:336-42.

Accepted 11 June 1990 